PROC. OF JSCE,

No. 252, AUG. 1976

\title{
NONLINEAR ANALYSIS OF SPATIAL FRAMES CONSISTING OF MEMBERS WITH CLOSED CROSS-SECTIONS
}

\author{
By Sadao KomatSU* and Tatsuro SAKIMOTO**
}

\section{SYNOPSIS}

A general matrix analysis for elasto-plastic spatial large deflections of thin-walled frames or arches is developed in this paper. A tangent stiffness matrix for an elasto-plastic member with closed cross-section and an initial stress matrix expressed in terms of internal forces are written with respect to so-called incremental moving coordinate. By the proposed method, the ultimate strength including buckling strength in bifurcation problems for arbitrary spatial frames or arches subjected to non-proportional loading can be analyzed under consideration of growth of plastic zones and initial imperfections such as residual stresses due to welding. Numerical examples are shown for the several problems which are presently available. Through the comparison with other authors' results, the accuracy and the efficiency of this method may be recognized.

\section{INTRODUCTION}

In such technically important structures as long span bridges, especially arch bridges, which are governed by compressive stresses, finite displacements and partial yielding phenomena have a profound effect on their static behavior. To ignore both, as is done in most instability analyses, invites major errors, usually on the unsafe side. Almost all studies for finite deflections have been so far confined to elastic problems, and the plastic analyses have been based on the popular plastic hinge theory associated with equilibrium situation in an infinitesimal excursion from its original configuration. The plastic hinge theory improved to satisfy the plastic flow conditions in the plastic hinges seems to be one of useful approaches, at present, for

* Dr. Eng., Professor of Civil Engineering, Osaka University

** M. of Eng., Lecturer of Applied Mechanics, Kumamoto University obtaining an approximation of the plastic failure load $^{1) ~ 3)}$. However, since the plastic hinge theory, which is originally developed for flexural members, cannot include the longitudinal stretch of plastic zones and elasto-plastic state of the crosssections, it may not give so good solutions for the structures composed of the members subjected to axial forces as for those composed of flexural members only. Existing elasto-plastic analyses, most of which are restricted to special planar structures under proportional loading, would be considerably complicated for complex structures even though they are based on some simplifying assumptions.

The aim of the present study is to develop a general matrix stiffness analysis for three dimensional elasto-plastic behavior accompanying finite deflections of thin-walled frames and arches with closed cross-sections. This method can also treat the ultimate strength, including buckling strength in bifurcation problems, for arbitrary spatial frames and arches subjected to non-proportional loading under consideration of growth of plastic zones, for which no method is presently available.

In the matrix stiffness method concerning geometrically nonlinear problems, a common technique is to obtain the equilibrium equations between total nodal forces and total nodal displacements from partial derivatives of the total potential energy in Lagrangian expression ${ }^{4}$. The stiffness matrices derived thus are usually described by the first order stiffness matrix corresponding to infinitesimal displacements and two additional matrices which contain respectively the linear and quadratic terms of unknown total nodal displacements. Solving this kind of equilibrium equations, unknown total nodal displacements will be uniquely found for the given nodal forces. This fact corresponds to Kirchhoff's theorem regarding uniqueness of solution in elastic problems.

On the contrary, as for the problems where the effects of the plasticity of a material should 
be taken into account, the equilibrium state will not be uniquely determined for a given set of total forces or total displacements except in the case of proportional loading, because the stress -strain relationships in the plastic range are generally dependent on the history of the strains or the stresses. Accordingly, the equilibrium equations cannot be described only by the total displacements and the total forces without introducing some complicated description denoting the strain history or the displacement paths. Then, the equilibrium equations expressed by the current stresses are needed. In this paper, the initial stress matrix expressed in terms of the current internal forces is introduced into the linear incremental equilibrium equations ${ }^{5}$. The equilibrium condition for a whole structural system is satisfied at each loading step between the given total external forces and the current internal forces calculated from the summations of incremental stresses.

Further difficulty is how to estimate the rigidities of members, especially the torsional rigidity of a partially yielded member subjected to combined forces ${ }^{6)}$ 11). The absence of an adequate way for the precise estimation of the torsional rigidities of partially yielded cross-sections seems to have hampered the theoretical development in the field of elasto-plastic analysis of spatial structures. In the present paper, these difficulties are overcome by extending the procedure reported in Ref. 11). By this procedure, the rigidities of elasto-plastic members can be estimated by numerical integrations, which are suitable for a digital computer by dividing the structural members into longitudinal finite elements and crosssectional sub-elements. The effects of initial residual stresses due to welding, which will play a very important role in the ultimate strength analysis, can be also easily taken into account by this procedure.

In the existing investigations, to the authors' knowledge, the analyses of the elasto-plastic behavior for non-proportional loading are very few. By applying the proposed method, the analysis concerning not only proportional loading but also non-proportional one can be performed by solving the equilibrium equations obtained herein step by step according to the given loading path.

\section{DERIVATION OF EQUILIBRIUM EQUATIONS}

\section{(1) Coordinate System and Assumptions}

A deformed body, of which strains are small although deflections and rotations may be large, will be described by a moving coordinate system fixed on the body $\left.{ }^{12}\right)^{-15}$ ). At first, let us consider a member element at an arbitrary reference state $i$ in the midst of some loading path. The local Cartesian coordinate system $(x, y, z)$ originated at the shear center $O$ of the cross section is set up and fixed to the element as illustrated in Figs. 1 and 2. The local coordinate system will be used as a Lagrangian frame for the subsequent state $i+1$. That is, the reference state $i$ may be considered as the initial stressed state for determining the stresses, strains and displacements of subsequent state $i+1$. A global coordinate system $(X, Y, Z)$ fixed to the space is used to assemble all the elements in the whole structural system.

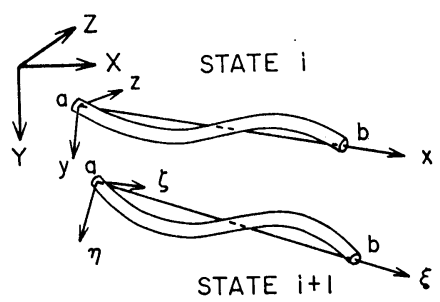

Fig. 1 Incremental moving coordinate system.

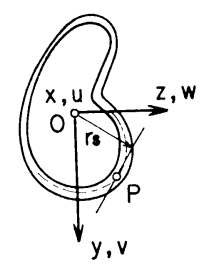

STATE i

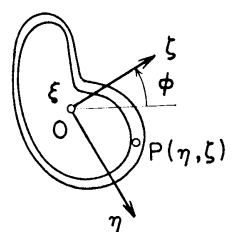

STATE $i+1$
Fig. 2 Incremental displacements and rotation of cross section.

This analysis is based on the following assumptions and idealizations:

1) The structure is composed of members with closed cross-section and made of elastic-perfectly plastic material.

2) The shape of the cross section and the dimension of the member are such that the effects of the warping torsion can be disregarded in comparison with those of the St. Venant torsion.

3) Bernuilli-Navier's hypothesis about bending strains is also valid even for a partially yielded cross-section.

4) The yield criterion of von Mises is acceptable 
and the stress-strain relationships of PrandtlReuss are valid in the plastic range.

5) The shear flows due to St. Venant torsion are uniformly distributed over the cross section with one cell, and the shear center may not change its location after partial yielding of the cross section.

6) The effects of the shear stress due to bending are small enough to be disregarded.

7) The strains are small although the displacements may be large.

8) Local buckling would not occur and the distortion of the cross-sectional shape is so small as to be negligible.

In the following formulations, the total displacements, strains, stresses and the total external forces are designated by the letters with bar over them and the ordinary letters without bar denote the increments of them, respectively.

\section{(2) The Relationships between Strains and Dis- placements}

The displacements $\left(\bar{u}_{P}, \bar{v}_{P}, \bar{w}_{P}\right)$ of an arbitrary point $P(\eta, \zeta)$ in any cross section will be expressed by the displacements $(\bar{u}, \bar{v}, \bar{w})$ of the shear center $O$ as well as the rotation $\bar{\phi}$ of the cross section as follows:

$$
\left.\begin{array}{rl}
\bar{u}_{P}= & \bar{u}-(\eta \cdot \cos \bar{\phi}-\zeta \cdot \sin \bar{\phi}) \cdot \bar{v}^{\prime} \\
& -(\eta \cdot \sin \bar{\phi}+\zeta \cdot \cos \bar{\phi}) \cdot \bar{w}^{\prime}+\bar{u}^{*}(\eta, \zeta) \\
\bar{v}_{P}= & \bar{v}-\eta \cdot(1-\cos \bar{\phi})-\zeta \cdot \sin \bar{\phi} \\
\bar{w}_{P}= & \bar{w}+\eta \cdot \sin \bar{\phi}-\zeta \cdot(1-\cos \bar{\phi}),
\end{array}\right\}
$$

where the superscript dash means differentiation with respect to $x$ and $\vec{u}^{*}(\eta, \zeta)$ is a longitudinal displacement due to warping. The relationship between the displacements and the normal strain is

$$
\begin{aligned}
\bar{\varepsilon}=\bar{u}_{P}^{\prime} & +\frac{1}{2}\left(\bar{u}_{P}^{\prime}\right)^{2}+\frac{1}{2}\left(\bar{v}_{P}^{\prime}\right)^{2}+\frac{1}{2}\left(\bar{w}_{P}^{\prime}\right)^{2} \\
& +(\text { higher order terms) } \\
\doteqdot & \bar{u}_{P}^{\prime}+\frac{1}{2}\left(\bar{v}_{P}^{\prime}\right)^{2}+\frac{1}{2}\left(\bar{w}_{P}^{\prime}\right)^{2} . \ldots \ldots \ldots . . .
\end{aligned}
$$

Substituting Eq. (1) into Eq. (2), the normal strain of an arbitrary point $P$ will be expressed by the displacements of the shear center as follows: ${ }^{16)}$

$$
\begin{aligned}
\bar{\varepsilon}= & \bar{u}^{\prime}-(\eta-\zeta \cdot \bar{\phi}) \cdot \bar{v}^{\prime \prime}-(\zeta+\eta \cdot \bar{\phi}) \cdot \bar{w}^{\prime \prime} \\
& +\frac{1}{2}\left\{\left(\bar{v}^{\prime}\right)^{2}+\left(\bar{w}^{\prime}\right)^{2}\right\}+\frac{1}{2}\left(\eta^{2}+\zeta^{2}\right)\left(\bar{\phi}^{\prime}\right)^{2} \ldots
\end{aligned}
$$

in which $\bar{u}^{*}$ is ignored according to the assumption 2) and the displacements remain up to the quadratic terms after the Maclaurin series expansion of the terms $\sin \bar{\phi}$ and $\cos \bar{\phi}$. On the other hand, the shear strain $\bar{\gamma}$ due to St. Venant torsion is given by the well-known formula ${ }^{17}$,

$$
\bar{\gamma}=r_{s} \bar{\phi}^{\prime}+\frac{\partial \bar{u}^{*}}{\partial s}
$$

where, the symbol $r_{s}$ denotes the distance from the shear center to a tangent drawn at any point on the middle line of the thin wall at the cross section and $s$ is a curvilinear coordinate along the middle line of the thin wall. If Eq. (3) is considered to be total Lagrangian expression ${ }^{15)}$, the strain increment would be given as follows:

$$
\begin{aligned}
\varepsilon= & u^{\prime}-(\eta-\zeta \cdot \bar{\phi}) \cdot v^{\prime \prime}-(\zeta+\eta \cdot \bar{\phi}) \cdot w^{\prime \prime} \\
& +\left(\zeta \cdot \bar{v}^{\prime \prime}-\eta \cdot \bar{w}^{\prime \prime}\right) \cdot \phi+\bar{v}^{\prime} \cdot v^{\prime}+\bar{w}^{\prime} \cdot w^{\prime} \\
& +\left(\eta^{2}+\zeta^{2}\right) \cdot \bar{\phi}^{\prime} \cdot \phi^{\prime} . \cdots \cdots \ldots \ldots \ldots \ldots \ldots \ldots \ldots \ldots \ldots \ldots
\end{aligned}
$$

But, in the present analysis, since the subsequent state $i+1$ is assumed to be incrementally close to the reference state $i$ and the reference frame will be gradually moved for each load step, the strain increments will be expressed in the form:

$$
\begin{aligned}
\varepsilon= & u^{\prime}-(\eta-\zeta \cdot \phi) \cdot v^{\prime \prime}-(\zeta+\eta \cdot \phi) \cdot w^{\prime \prime} \\
& +\frac{1}{2}\left\{\left(v^{\prime}\right)^{2}+\left(w^{\prime}\right)^{2}\right\}+\frac{1}{2}\left(\eta^{2}+\zeta^{2}\right)\left(\phi^{\prime}\right)^{2}
\end{aligned}
$$

and

$$
\gamma=r_{s} \cdot \phi^{\prime}+\frac{\partial u^{*}}{\partial s}
$$

When the displacement increments are small, the normal strain increment is given by the ordinary linear equation. That is,

$$
\varepsilon=u^{\prime}-\eta \cdot v^{\prime \prime}-\zeta \cdot w^{\prime \prime}
$$

\section{(3) Tangent Stiffness Matrix of Partially Yielded Closed Cross-Sections ${ }^{11}$ )}

The relationships between the increments of the internal forces $\left(N, M_{\xi}, M_{\eta}, M_{\zeta}\right)$ and the deformation increments $\left(\varepsilon_{0}, \phi^{\prime}, \phi_{\eta}, \phi_{\zeta}\right)$ should be derived about a partially yielded closed cross-section of unit length as illustrated in Fig. 3. The stress-

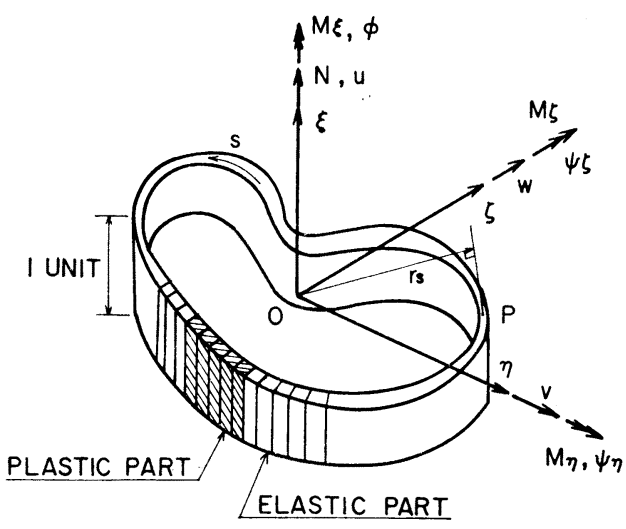

Fig. 3 Partially yielded cross-section. 
strain relationships in the plastic range may be given by the Prandtl-Reuss equations as follows:

$$
\gamma=\frac{\tau}{G}+\frac{3 \bar{\tau}}{\bar{\sigma}}\left(\varepsilon-\frac{\sigma}{E}\right)
$$

and

$$
\sigma=-\frac{3 \bar{\tau}}{\bar{\sigma}} \cdot \tau,
$$

where $\bar{\sigma}$ and $\bar{\tau}$ are normal stress and shear stress respectively and $E$ and $G$ are Young's modulus and shear modulus of elasticity, respectively. In the elastic range, since Hooke's law is acceptable,

$$
\gamma=\frac{\tau}{G}
$$

and

$$
\sigma=E \cdot \varepsilon .
$$

Performing the closed linear integrals of Eq. (6) over the whole cross-section after substituting Eq. (7) for the plastic zones and Eq. (9) for the elastic zones into Eq. (6) yields the following equation:

$$
\begin{array}{r}
\oint \frac{\partial u^{*}}{\partial s} d s+\phi^{\prime} \oint r_{s} d s=\frac{1}{G} \int_{e} \tau d s+\frac{1}{G} \int_{p} \tau d s \\
-\frac{1}{E} \int_{p} \frac{3 \bar{\tau}}{\bar{\sigma}} \sigma d s+\int_{p} \frac{3 \bar{\tau}}{\bar{\sigma}} \varepsilon d s, \cdots \cdots \cdots(11)
\end{array}
$$

in which $\oint d s$ denotes closed linear integral and $\int_{e} d s$ and $\int_{p} d s$ are linear integrals over the elastic zones and plastic zones, respectively. The first term on the left hand side of Eq. (11) will vanish, because the warping of a closed crosssection must be continuous at any point. Substituting Eqs. $\left(5^{\prime}\right)$ and (8) into Eq. (11) and solving it with respect to the shear flow $q=\tau \cdot t$ yield the following equation:

$$
q=\frac{-1}{C_{1}+C_{2}}\left(C_{3} \varepsilon_{0}-C_{4} \psi_{6}+C_{5} \phi_{\eta}+C_{6} \theta\right) \cdots
$$

in which

$$
\begin{array}{ll}
\varepsilon_{0}=u^{\prime}, \quad \phi_{\zeta}=v^{\prime \prime}, & \phi_{\eta}=-w^{\prime \prime}, \quad \theta=\phi^{\prime}, \\
C_{1}=\frac{1}{G} \oint \frac{d s}{t}, & C_{2}=\frac{1}{E} \int_{p}\left(\frac{3 \bar{\tau}}{\bar{\sigma}}\right)^{2} \frac{d s}{t}, \\
C_{3}=\int_{p} \frac{3 \bar{\tau}}{\bar{\sigma}} d s, & C_{4}=\int \frac{3 \bar{\tau}}{\bar{\sigma}} \eta d s, \\
C_{5}=\int_{p} \frac{3 \bar{\tau}}{\bar{\sigma}} \zeta d s, & C_{6}=-\oint r_{s} d s .
\end{array}
$$

On the other hand, the increments of the internal forces may be expressed by the integration of stress increments over the cross-sectional area. That is,

$$
\begin{aligned}
& N=E \int_{e} \varepsilon d A-\int_{p} \frac{3 \bar{\tau}}{\bar{\sigma}} \tau d A, \\
& M_{\zeta}=-E \int_{e} \varepsilon \eta d A+\int_{p} \frac{3 \bar{\tau}}{\bar{\sigma}} \tau \eta d A, \\
& M_{\eta}=E \int_{e} \varepsilon \zeta d A-\int_{p} \frac{3 \bar{\tau}}{\bar{\sigma}} \tau \zeta d A, \\
& M_{\xi}=\oint \tau r_{s} d A .
\end{aligned}
$$

Substituting Eqs. (5') and (12) into Eq. (14) yields the tangent stiffness matrix for a closed crosssection as follows:

$$
\boldsymbol{f}=\left[\boldsymbol{S}_{e}+\boldsymbol{S}_{p}\right] \cdot \boldsymbol{d}
$$

in which

$$
\begin{aligned}
& \boldsymbol{f}=\left[\begin{array}{lll}
N-M_{\zeta} & M_{\eta} & M_{\xi}
\end{array}\right]^{T}, \quad \boldsymbol{d}=\left[\begin{array}{lll}
\varepsilon_{0}-\phi_{\zeta} & \phi_{\eta} & \theta
\end{array}\right]^{T}, \\
& \boldsymbol{S}_{e}=\left(\begin{array}{rrrr}
E A_{e} & E S_{\zeta} & E S_{\eta} & 0 \\
E I_{\zeta} & E I_{\eta \zeta} & 0 \\
& E I_{\eta} & 0 \\
\text { sym. } & & G I_{\xi}
\end{array}\right), \boldsymbol{S}_{p}=\left(\begin{array}{cccc}
D_{1} & D_{2} & D_{3} & D_{4} \\
D_{5} & D_{6} & D_{7} \\
& D_{8} & D_{9} \\
\text { sym. } & & D_{10}
\end{array}\right), \\
& A_{e}=\int_{e} d A, \quad S_{\zeta}=\int_{e} \eta d A, \quad S_{\eta}=\int_{e} \zeta d A, \\
& I_{\zeta}=\int_{e} \eta^{2} d A, \quad I_{\eta \zeta}=\int_{e} \eta \zeta d A, \quad I_{\eta}=\int_{e} \zeta^{2} d A, \\
& I_{\xi}=\left(\oint r_{s} d S\right)^{2} / \oint \frac{1}{t} d S \text {, } \\
& D_{1}=\frac{C_{3}^{2}}{C_{1}+C_{2}}, \quad D_{2}=\frac{C_{3} C_{4}}{C_{1}+C_{2}}, \quad D_{3}=\frac{C_{3} C_{5}}{C_{1}+C_{2}}, \\
& D_{4}=\frac{C_{3} C_{6}}{C_{1}+C_{2}}, \quad D_{5}=\frac{C_{4}^{2}}{C_{1}+C_{2}}, \quad D_{6}=\frac{C_{4} C_{5}}{C_{1}+C_{2}}, \\
& D_{7}=\frac{C_{4} C_{6}}{C_{1}+C_{2}}, \quad D_{8}=\frac{C_{5}^{2}}{C_{1}+C_{2}}, \quad D_{9}=\frac{C_{5} C_{6}}{C_{1}+C_{2}}, \\
& D_{10}=\frac{-C_{2} C_{6}^{2}}{C_{1}\left(C_{1}+C_{2}\right)} \text {. }
\end{aligned}
$$

In Eq. (15), the matrices $\boldsymbol{S}_{e}$ and $\boldsymbol{S}_{\boldsymbol{p}}$ consist of the quantities related to elastic zones and plastic zones, respectively. The matrix $\boldsymbol{S}_{p}$ will not appear for an entirely elastic cross-section and only the square matrix $\boldsymbol{S}_{e}$, which has only four diagonal nonzero terms for a doubly symmetric cross-section, remains. For a fully plastic cross -section, only the matrix $\boldsymbol{S}_{p}$ will remain and hold the flow conditions of plasticity between $\boldsymbol{f}$ and $\boldsymbol{d}$.

\section{(4) Tangent Stiffness Matrix of a Member Element}

An incremental tangent stiffness matrix may be derived by applying the incremental variational method $^{18)}$ commonly used in the finite element formulations. When a member, starting from 
the initial state $i$ prestressed under total external loads $\overline{\boldsymbol{p}}$, reaches to the subsequent state $i+1$ after producing increments of displacement due to load increments $\boldsymbol{p}$, the incremental potential energy $V$ during deformation may be given as follows:

$$
\begin{aligned}
V & =-\left\{(\overline{\boldsymbol{u}}+\boldsymbol{u})^{T}(\overline{\boldsymbol{p}}+\boldsymbol{p})-\overline{\boldsymbol{u}}^{T} \boldsymbol{p}\right\} \\
& =-\boldsymbol{u}^{T}(\overline{\boldsymbol{p}}+\boldsymbol{p})-\overline{\boldsymbol{u}}^{T} \boldsymbol{p}, \cdots \cdots
\end{aligned}
$$

in which the superscript $T$ stands for the transposition of the matrix. Assuming the linearity during the incremental step, the incremental strain energy $U$ stored in the element of length $l$ is:

$$
\begin{aligned}
U= & \frac{1}{2} \int_{0}^{l} \boldsymbol{d}^{T} \boldsymbol{f} \cdot d x+\int_{0}^{l}\left(\int_{A} \bar{\sigma} \varepsilon d A\right) d x \\
& +\int_{0}^{l} \bar{M}_{\xi} \phi^{\prime} d x .
\end{aligned}
$$

Substituting Eqs. (5) and (15) into Eq. (18) yields

$$
\begin{aligned}
U= & \frac{1}{2} \int_{0}^{l} \boldsymbol{d}^{T}\left(\boldsymbol{S}_{e}+\boldsymbol{S}_{p}\right) \boldsymbol{d} \cdot d x \\
& +\int_{0}^{l} \bar{N}\left\{u^{\prime}+\frac{1}{2}\left(v^{\prime}\right)^{2}+\frac{1}{2}\left(w^{\prime}\right)^{2}\right\} d x \\
& +\int_{0}^{l} \bar{M}_{\zeta}\left(v^{\prime \prime}+\phi w^{\prime \prime}\right) d x-\int_{0}^{l} \bar{M}_{\eta}\left(w^{\prime \prime}-\phi v^{\prime \prime}\right) d x \\
& +\frac{1}{2} \int_{0}^{l} \bar{T}\left(\phi^{\prime}\right)^{2} d x+\int_{0}^{l} \bar{M}_{\xi} \phi^{\prime} d x \quad \cdots \cdots(19)
\end{aligned}
$$

in which

$$
\begin{array}{ll}
\bar{N}=\int_{A} \bar{\sigma} d A, \quad \bar{M}_{\zeta}=-\int_{A} \bar{\sigma} \eta d A, \\
\bar{M}_{\eta}=\int_{A} \bar{\sigma} \zeta d A, \quad \bar{T}=\int_{A} \bar{\sigma}\left(\eta^{2}+\zeta^{2}\right) d A, \\
\bar{M}_{\xi}=\oint \bar{\tau} r_{s} d A .
\end{array}
$$

Since no information is available for the displacement functions of an elasto-plastic finite element, polynomials, which are usually used for an elastic finite element analysis, are also used herein. That is, $u, \phi$ and $v, w$ are approximated by linear and cubic polynomials of $x$, respectively. Internal forces $\bar{N}, \bar{T}$ and $\bar{M}_{\xi}$ are also assumed to be uniform along $x$ and $\bar{M}_{\zeta}$ and $\bar{M}_{\eta}$ are assumed to be linear polynomials of $x$. Then, introducing the usual technique used in the finite element method ${ }^{4}$, Eq. (19) can be expressed in terms of incremental nodal displacements and internal forces at nodal point. Finally, the increment of the potential energy $\pi=U+V$ can be described by the increments of the nodal displacement $\boldsymbol{u}$. Since the stationary condition of $\pi$ gives the incremental equilibrium condition, first partial derivatives of $\pi$ with respect to $\boldsymbol{u}$ will yield the following incremental equilibrium equations:

$$
\left(\boldsymbol{k}_{e}+\boldsymbol{k}_{\boldsymbol{p}}+\boldsymbol{k}_{g}\right) \cdot \boldsymbol{u}=\boldsymbol{p}-(\overline{\boldsymbol{f}}-\overline{\boldsymbol{p}})
$$

in which the displacement vector and forces vectors are given as follows:

$$
\begin{aligned}
& \boldsymbol{u}=\left[u_{a} v_{a} w_{a} \phi_{a} \alpha_{a} \beta_{a} u_{b} v_{b} w_{b} \phi_{b} \alpha_{b} \beta_{b}\right]^{T}, \\
& \alpha=-w^{\prime}, \quad \beta=v^{\prime}, \\
& \boldsymbol{p}=\left[P_{x a} P_{y a} P_{z a} M_{x a} M_{y a} M_{z a}\right. \\
& \left.P_{x b} P_{y b} P_{z b} M_{x b} M_{y b} M_{z b}\right]^{T}, \\
& \overline{\boldsymbol{p}}=\left[\bar{P}_{x a} \bar{P}_{y a} \bar{P}_{z a} \bar{M}_{x a} \bar{M}_{y a} \bar{M}_{z a}\right. \\
& \left.\bar{P}_{x b} \bar{P}_{y b} \bar{P}_{z b} \bar{M}_{x b} \bar{M}_{y b} \bar{M}_{z b}\right]^{T}, \\
& \overline{\boldsymbol{f}}=\left[-\frac{\bar{N}_{a}+\bar{N}_{b}}{2}-\frac{\bar{M}_{\zeta a}-\bar{M}_{\zeta b}}{l} \frac{\bar{M}_{\eta a}-\bar{M}_{\eta b}}{l}\right. \\
& -\frac{\bar{M}_{\xi a}+\bar{M}_{\xi b}}{2}-\bar{M}_{\eta a}-\bar{M}_{\zeta a} \\
& \frac{\bar{N}_{a}+\bar{N}_{b}}{2} \frac{\bar{M}_{\zeta a}-\bar{M}_{\zeta b}}{l} \\
& \left.-\frac{\bar{M}_{\eta a}-\bar{M}_{\eta b}}{l} \frac{\bar{M}_{\xi a}+\bar{M}_{\xi b}}{2} \bar{M}_{\eta b} \bar{M}_{\zeta b}\right]^{T} \text {. }
\end{aligned}
$$

In the above equations (22), the suffixes $a$ and $b$ denote the quantities related to both the ends of an element. The stiffness matrices $\boldsymbol{k}_{e}, \boldsymbol{k}_{\boldsymbol{p}}$ and $\boldsymbol{k}_{g}$ are obtained as shown in Appendix 1. The matrix $\boldsymbol{k}_{e}$ represents the stiffness of elastic part and the matrix $\boldsymbol{k}_{p}$ the reduction of stiffness due to yielding of the material. The matrix $\boldsymbol{k}_{g}$ is the initial stress matrix including nodal internal forces of the element at the initial state $i$. In Eq. (21), the equation $\overline{\boldsymbol{f}}-\overline{\boldsymbol{p}}=0$ means that a total equilibrium condition is satisfied. Accordingly, the term $(\overline{\boldsymbol{f}}-\overline{\boldsymbol{p}})$ will be considered as the unbalanced forces ${ }^{13)}$ owing to the linearization in the formulation and the yielding of the material in the midst of a step of incremental loading. In order to eliminate these unbalanced forces, the iterative procedure stated later will be needed. Transformation of Eq. (21) to the global coordinate system by the transformation matrix ${ }^{19}$ ) $\boldsymbol{T}$ yields:

$$
\left[\boldsymbol{K}_{e}+\boldsymbol{K}_{p}+\boldsymbol{K}_{g}(\overline{\boldsymbol{f}})\right] \cdot \boldsymbol{U}=\boldsymbol{P}-(\boldsymbol{T} \cdot \overline{\boldsymbol{f}}-\overline{\boldsymbol{P}}) \ldots
$$

An equation constructed by assembling Eq. (23) for a whole structural system should be solved after all. Such equation has, of course, an identical form with that of Eq. (23).

\section{PROCEDURE FOR SOLUTION}

\section{(1) Computation Procedure}

In order to trace the growth of plastic zones both along the member axis and through the 
cross section, the structure is divided into a number of member elements and cross sectional sub-elements. The strains and stresses of each element are evaluated at the centroids of the cross sectional sub-elements. Cross sectional quantities and internal forces appearing in the stiffness matrix are evaluated by a numerical integration at both the ends of the member element. Since Bernuilli-Navier's hypothesis constrains the movement between mutual subelements, the degree of freedom of the displacements may be reduced effectively and the size of the matrix to be solved depends on only the number of the nodal points. Generally speaking, in order to improve the accuracy about the estimation of the plastic zones, it is needed to divide the structure into much more member elements than the elements for an ordinary elastic analysis. The numerical integration covering the sub-elements make it possible to analyze the complex structures composed of arbitrary closed cross-sections with arbitrary residual stress distributions.

\section{(2) Solution Procedure}

The Newton-Raphson method is well suited for certain types of nonlinear analysis and usually used in the analyses of nonlinear elastic problems ${ }^{20)}$. Not only the incremental equilibrium equations, but also the total equilibrium equations are needed for the Newton-Raphson method. Since, in the present formulation, the total equilibrium equations can be obtained by an incremental procedure in the same manner as elastic problems, in spite of the plastic problems, which are dependent on the history, the Newton-Raphson method can be employed in this analysis too. Since the Newton-Raphson method is considered to be an incremental procedure with a selfcorrecting process ${ }^{15)}$, it seems to permit comparatively large load increments and it is true, to some extent, for the elastic analysis based on the Lagrangian coordinate approach. In the present method, as described in the former chapter, since the subsequent loading state $i+1$ is assumed to be incrementally close to the reference state $i$, too large load increments i.e. too large displacement increments may lead the numerical errors peculiar to the moving coordinate approach even for the elastic problems ${ }^{5}$. Furthermore, for the plastic problems, especially for the cases heavily influenced by the history, it is necessary to give enough small load increments to obtain the results of satisfactory accuracy. One of the method to control the load increments is to give the load increments so as to take place the yielding in only one sub-element at one load step $^{21)}$, but the computational efforts will increase remarkably. Though it is needed to determine an appropriate size of load increments by considering both the required accuracy of the solution and the costs of computation, it is difficult to seek for an optimum and general method controlling the load increments for various structures of various behaviors. In the present analysis, particular load control process is not used because of above mentioned reason, but the numerical examples given in the next chapter show that the present method will give fairly good results in spite of comparatively large load increments. The process of numerical calculation is illustrated in the flow chart given in Appendix 2, and may be summarized as follows:

1) Compute the displacement increments $U$ due to load increments $\boldsymbol{P}$ referred to global coordinate system.

2) Evaluate the stresses of each cross-sectional sub-elements at both the ends of member elements and check the yielding. Compute new cross sectional quantities of the members from the averages of the corresponding quantities of their both ends.

3) Renew the coordinates of the nodal points and revise the transformation matrices $T$.

4) Calculate the internal forces $\overline{\boldsymbol{f}}$ as resultants of accumulated stresses and compute the unbalanced forces $(\boldsymbol{T} \overline{\boldsymbol{f}}-\overline{\boldsymbol{P}})$.

5) Compose the new stiffness matrices $\boldsymbol{K}_{e}, \boldsymbol{K}_{p}$ and $\boldsymbol{K}_{g}$.

6) Give next load increments if the specified convergence criteria are satisfied just after the iterative calculation from 1) to 5).

7) Stop the computation if trivial large displacements due to buckling would take place or a mechanism condition would be satisfied.

\section{EXAMPLE PROBLEMS}

A number of typical numerical examples are dealt with for demonstrating the rationality and efficiency of the proposed method.

\section{(1) Compressive Member of Initial Imperfections}

The load-deflection diagrams of a column with initial deflection and residual stresses are illustrated in Fig. 4. The curve given by elastic analysis, where yielding of the material is disregarded, approaches assymptotically the straight line corresponding to Euler's buckling load, and the critical loads of plastic analysis show good agreement with the values given by Shul $z^{23)}$. It 
may be recognized from the shapes of the curves by the plastic analysis that the column with box cross section as illustrated here is weaken abruptly due to sudden total yielding of the flange plate of the concave side of the deformed column. It is also found from this example that even such a small number of section elements as 24 gives practically enough accuracy.

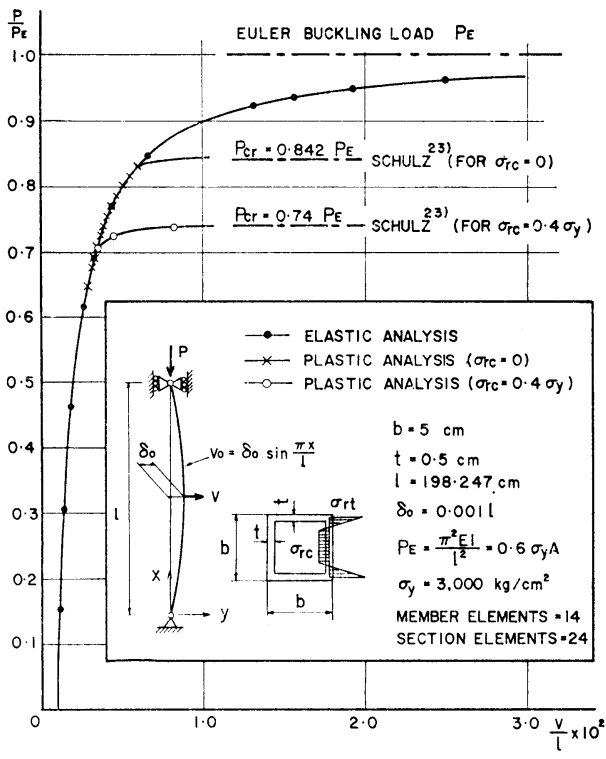

Fig. 4 Column with initial imperfections.

\section{(2) Deep Arch with a Concentrated Load}

A pin-ended semicircular arch under a concentrated load at the crown is treated here. In -plane elastic buckling and post buckling behavior of circular arches of this type have been investigated analytically by Dadeppo-Schmidt by means of an elliptical integration ${ }^{24)}$. This example is taken up here so as to study mainly the effects of the following two subjects on the accuracy of the results. One is the number of member elements and the other is the substitution of a polygonal structure composed of straight finite elements for an actual curved structure. In the calculation for this example, the number of iteration at load step $n$ is given as $3 \times n$ in each step. Fig. 5(a) shows the applied load-central deflection diagram. In this figure, the results of the present method, in spite of fairly large load increments, show good coincidence with the results of Ref. 24) until the load attains to the critical buckling load $p_{c r}=5.86$. The deflected configuration at the load $p=5.83$ is shown in Fig. $5(\mathrm{~b})$. It is seen from this figure that the present method

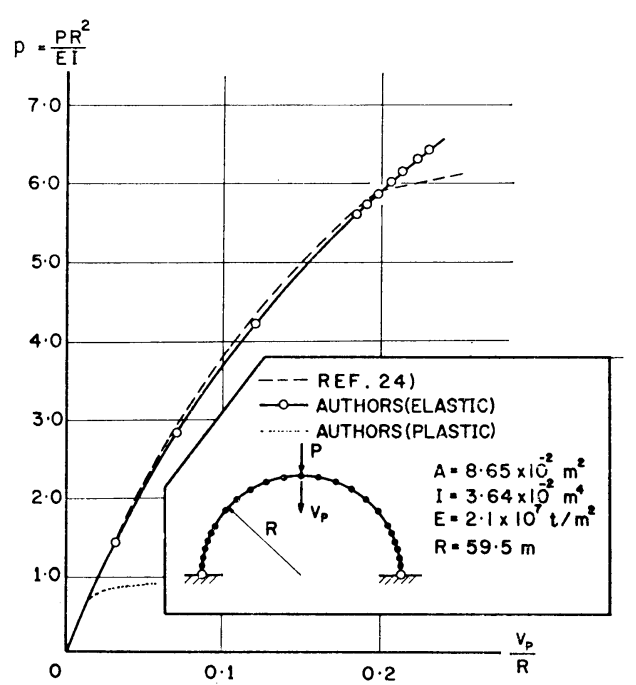

Fig. 5(a) Load versus central deflection diagrams of semicircular arch.

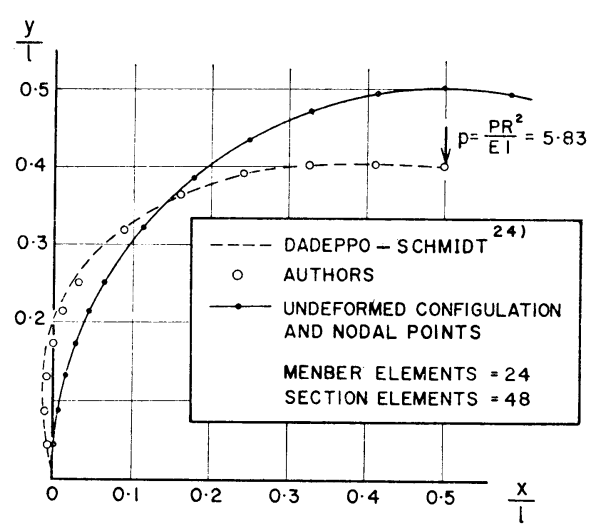

Fig. 5(b) Deformed configuration of semicircular arch at $p=5.83$.

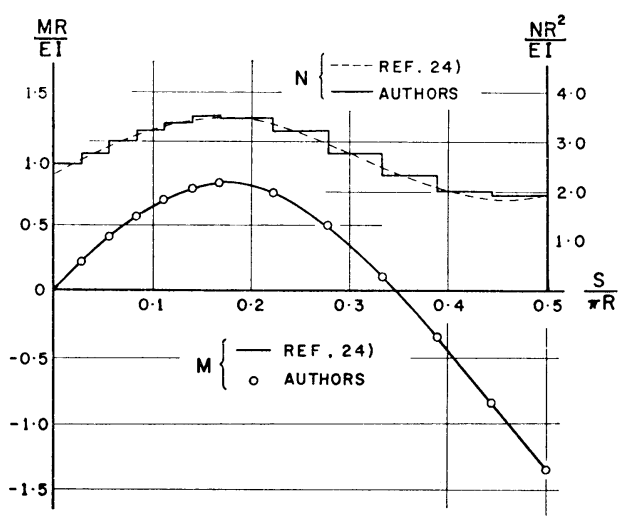

Fig. 5(c) Moment and thrust diagram of semicircular arch at $p=5.83$. 


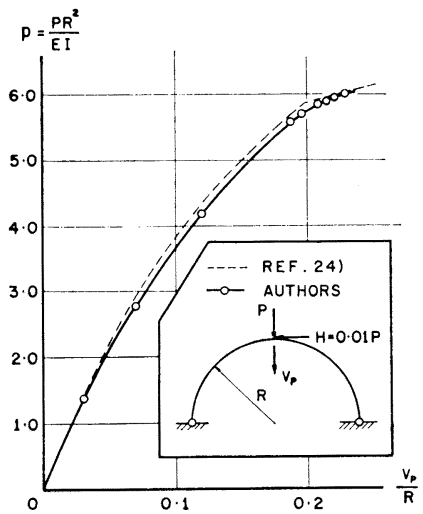

Fig. 5(d) Load versus central deflection diagrams of semicircular arch with a horizontal force as an initial disturbance.

is effective enough for a remarkably nonlinear problem like this. The variation of the bending moment and axial thrust at the load $p=5.83$ is also illustrated in Fig. 5(c). As for the values of bending moment, the results of this method coincide with those of Ref. 24) very well. The axial thrust diagram in Fig. 5(c) shows stepwise variation and is discontinuous at the nodal points, because the solutions of the present method are found for the idealized polygonal frame. However, if the values at the mid-points of the member elements are considered as the actual ones, the results fairly correspond with the curve of Ref. 24). It is possible, of course, to make the differences of the stepwise variation more small by dividing the structure into much more member elements. The inclination of the load-deflection diagram of Ref. 24) in Fig. 5(a) varies abruptly just after the load attains to the critical load of sidesway buckling. In the present method, it is also possible to analyze such nonlinear buckling producing large deflection as this example by introducing small initial imperfections into the structure as shown in the previous example (1). For example, the load-central deflection diagram which is obtained under the vertical load $P$ together with the horizontal load $H=0.01 P$ as an initial disturbance at the crown, fairly corresponds to the one of Ref. 24) as illustrated in Fig. 5(d). An asymmetrical initial deflection also may be introduced as an initial infinitesimal disturbance for sidesway buckling. These techniques would be rather equivalent to the procedure in the analysis of bifurcation problems in which the equilibrium equations will be formulated for an

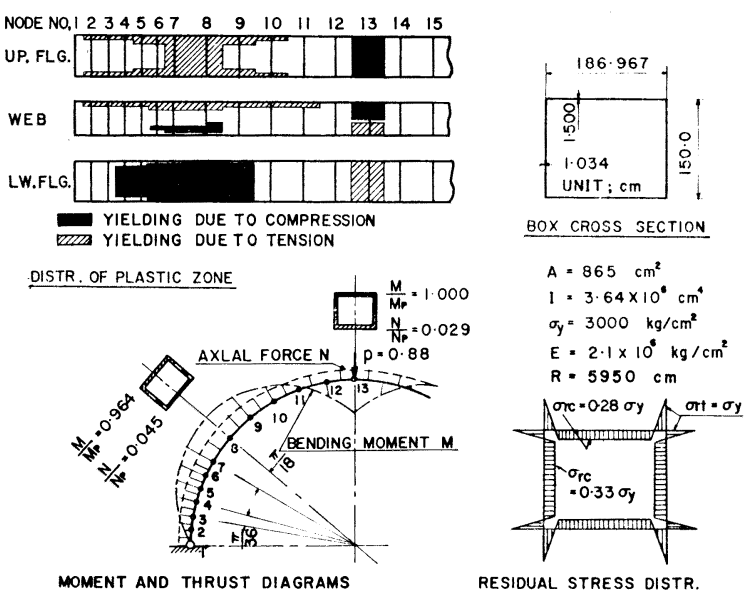

Fig. 6 Inelastic analysis of semicircular arch.

infinitesimally deflected configulation. The arches in Fig. 5 are also assumed to be made of idealized elastic material. If the arch is made of more realistic material like steel, yielding will occur before such a large deflection takes place. The results of inelastic analysis shown in Fig. 6 are about the arch identical with the one in Fig. 5 except considering the residual stresses due to welding. From the distribution of plastic zones, it will be recognized that the cross section at the quarter-points and the crown become almost fully plastic state and the arch is just before the failure due to a mechanism. The load-central deflection curve in this case, which is also illustrated in Fig. 5 (a) by a dotted line, shows that the rigidity of the arch is reduced very much at the load $p=0.9$ and large deflections are produced. It is noteworthy that the failure load in this case is only about $15 \%$ of the critical load for elastic sidesway buckling.

\section{(3) Parabolic Arch under Distributed Vertical Loads}

Pin-ended parabolic arches with rectangular cross-sections subjected to distributed vertical loads are studied here. Some results obtained by the present method are compared with those of Harries ${ }^{25)}$ and Kuranishi- $\mathrm{Lu}^{26)}$. The results of the present method are calculated for the frame whose configuration is an inscribed polygon in the given parabolic configuration, and the distributed loads are substituted into thirteen concentrated loads applied at the nodal points as shown in the inset of Fig. 7(b). The maximum loads of arches are plotted against the slenderness 
ratios for two loading cases in Fig. 7 (a). The results of the present analysis show good agreement with Harries' values. The values by Kuranishi-Lu seem to be $10 \sim 20 \%$ less than those by Harries or by authors in the case of $s=0.99$. One of the load-deflection diagram for the quarter -point are illustrated in Fig. 7(b) together with those by Kuranishi-Lu. The maximum loads given by the present method are about $16 \%$ larger than those of Kuranishi-Lu for $s=0.99$ and $7 \%$ for $s=0.5 .^{*}$

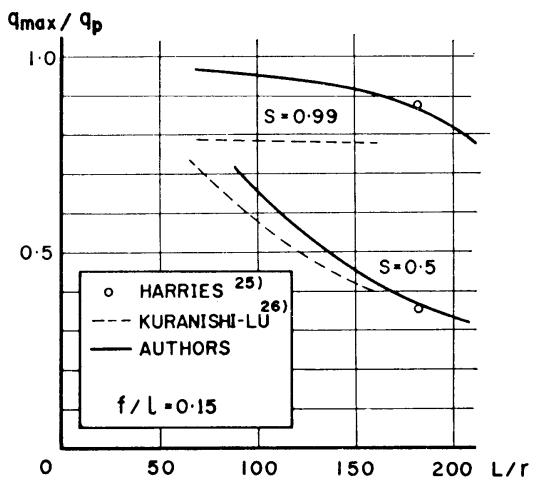

Fig. 7 (a) Maximum loads versus slenderness ratios diagram of pin-ended parabolic arches.

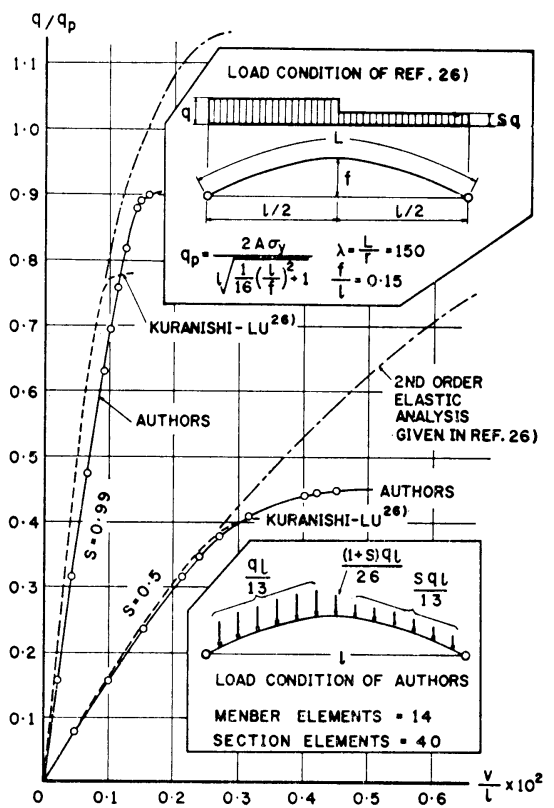

Fig. 7(b) Load-deflection diagram at quarter -point of pin-ended parabolic arches.

\section{(4) A Right-Angle Bent under a Concentrated Load}

A right-angle bent under a concentrated load is taken up as a simple spatial problem to examine the rationality of the present method for a frame subjected to combined torsion and bending. Ueda et al. have also investigated such a problem by a modified plastic hinge theory and estimated the plastic failure load through

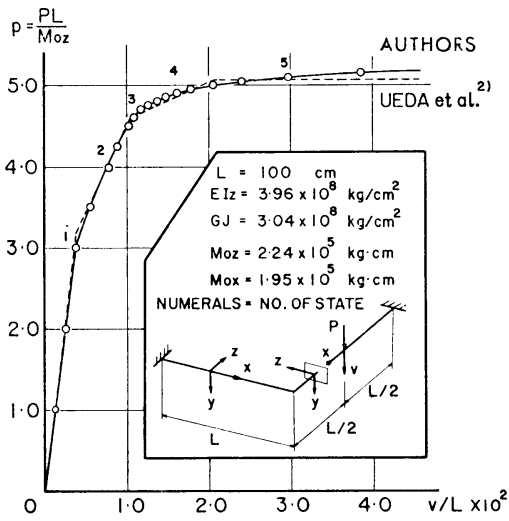

Fig. 8(a) Load-deffection diagram of right -angle bent.

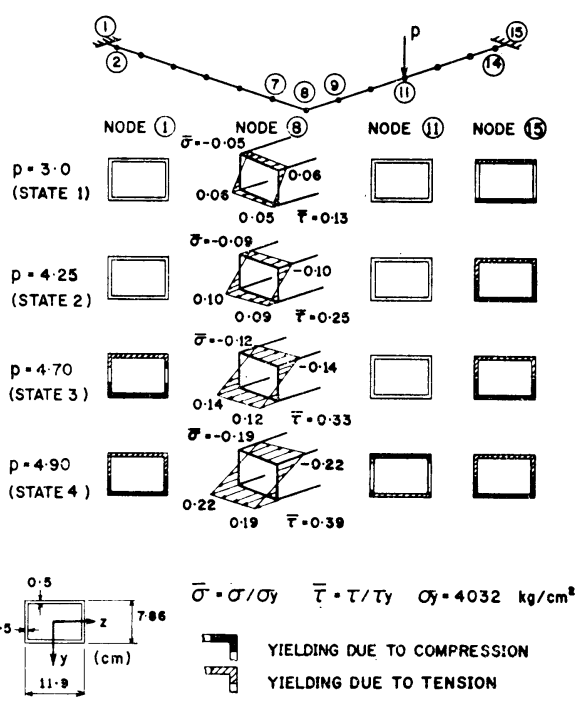

Fig. 8(b) Spread of plastic zones of right -angle bent.

* The results of present method for this case are compared with the results of recently published paper of Shinke et al. ${ }^{28)}$ and recognized to show good coincidence ${ }^{29)}$. 
tracing a forming process of plastic hinges ${ }^{2)}$. Fig. 8(a) shows the load-deflection diagram at the loading point of the beams. Since in the theory of Ref. 2) yielding of the material is limited to isolated plastic hinges and the shape factor of the cross section is inevitablly assumed to be unity, the load-deflection diagram obtained there is a folded straight line as illustrated by the broken like in Fig. 8(a). The present method, which can consider the spread of the plastic zones both along longitudinal direction and through the cross section as illustrated in Fig. 8(b), yields a smoothly continuous load-deflection diagram. Both curves are show fairly good correspondence except the difference of shape caused by the difference of the both methods stated above. In authors' calculation, a box cross section is chosen and determined to have the same cross-sectional properties as those in Ref. 2), but the shape

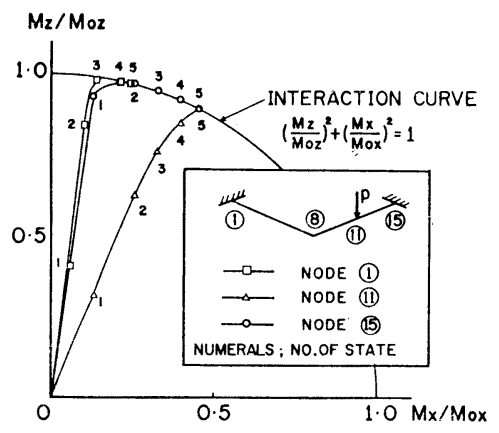

Fig. 8 (c) Variation of internal forces at the nodal points 1,11 and 15 of right -angle bent.
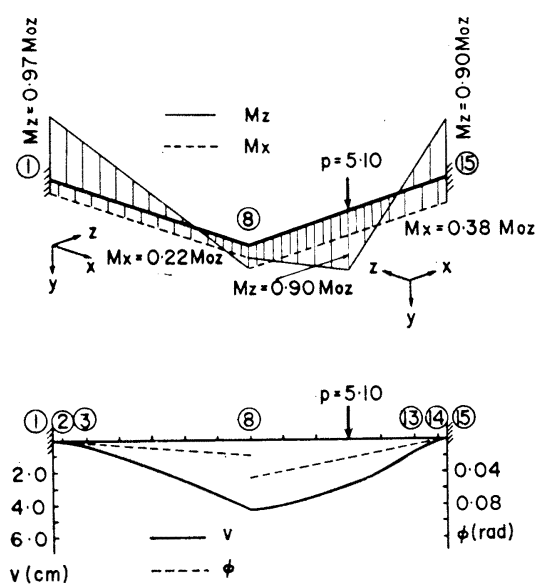

Fig. 8(d) Moment and deformation diagram of right-angle bent at the state 5 . factor has been taken equal to 1.08 as it is, without the idealization of making it unity. The difference of the failure load between the present result and that of Ref. 2) will be mainly caused by the difference of the shape factors. The collapse load of simple plastic hinge theory is computed by J. Heyman ${ }^{27)}$ as $p=5.1$ for an idealized box cross section. Ueda et al. have extended their theory to a finite displacement analysis, but the results for this example belong to a small deflection theory. The geometrical nonlinearity of this example will be small. The variation of the internal forces at the nodal points, which will become a fully plastic state, are shown in Fig. 8(c). It is seen from this figure that the internal forces vary along the yield surface after the cross section attains to a fully plastic state. The longitudinal variation of deflection $v$ and torsional angle $\phi$ are shown together with the moment diagram in Fig. 8(d). From these results, it will be recognized that the results of the present method will coincide with those of the plastic hinge theory as for the plastic analysis of the framed structures which will collapse without the longitudinal spread of the plastic zones like this example.

\section{CONCLUDING REMARKS}

A general matrix method for the problems including both geometric and material nonlinearity is presented. By using the proposed method, the spatial elasto-plastic behavior and ultimate strength of various framed structures can be analyzed in sufficient accuracy except for such problems as the elastica ${ }^{22}$ in which finite strains must be considered. Since this method is developed by introducing the elasto-plastic tangent stiffness matrix of closed cross-section proposed in Ref. 11) into the matrix method for geometrically nonlinear problems, it has many advantages which are proper to the matrix stiffness analysis. In the existing method for dealing with the problems covered in this paper, it is needed to perform individual formulation for each special structural system and boundary conditions and also to derive complex yield conditions for various shapes of cross sections individually. In authors' method, there is no necessity to do so and it is possible to analyze systematically arbitrary structural systems with various boundary conditions and various shapes of closed cross-sections only by changing the input data in the computer program.

Strictly speaking, when the shape of the cross section is other than square or circular ones, the 
effects of warping must be considered. As is stated in the assumption, the effects of warping are disregarded in this paper, but the solutions of the present method may give one of good approximations of ultimate strength even for the structures composed of the members whose cross -sectional shape is rather flat. As for the ultimate strength analyses of the spatial structures composed of the members of open cross-section, it will be necessary to clarify the phenomena about the warping torsion in the elasto-plastic range, since the warping torsion will become more important than the St. Venant torsion in such structural members. The knowledges about the warping torsion in the elasto-plastic range is not presently available, and it is not so usual case to use members of open cross-section for main members which should resist to torsional force.

In this paper, only the outline of the formulation and limited numerical examples are presented. But, it is supposed that developing and improving this method will open up the possibility to analyze quite a few unsolved problems.

\section{ACKNOWLEDGEMENTS}

Part of this investigation was supported by the Funds of Aid for Scientific Researches from the Ministry of Education. Thanks are due to Messrs. K. Kobayashi, S. Sugimoto and Y. Miyazaki for the help in preparing the computer programs. Numerical computations were carried out mainly by FACOM 230-75 computer of Kyushu University.

\section{REFERENCES}

1) Bruinette, K. E. and S. J. Fenves: A general formulation of the elasto-plastic analysis of space frameworks, papers presented at the International Conference on Space Structures, at University of Surrey, pp. 1-16, 1966.

2) Ueda, Y., T. Yamakawa and T. Akamatsu: A new theory on elastic-plastic analysis of framed structures, Technology Reports of the Osaka University, No. 875, Vol. 13, pp. 263-276, 1968.

3) Morino, S.: Elastic-plastic analysis of space frames, Bulletin of the Disaster Prevention Research Institute, Kyoto University, pp. 129, Vol. 15B, 1972. (in Japanese)

4) for example, Martin, H. C.: On the derivation of stiffness matrices for the analysis of large deflection and stability problems. Proc. of the 1-st International Conf. on Matrix Method in Structural Mech., pp. 697-726, Oct., 1965. Martin, H. C.: Finite elements and analysis of geometrically nonlinear problems, Paper presented at the U.S.-Japan Seminar held at Tokyo, Aug., 1969. Recent Advances in Matrix Method of Structural Analysis and Design, pp. 343-381, UAH Press, 1971.

5) Bažant, Z. P. and M. E. Nimeiri: Large -deflection spatial buckling of thin-walled beams and frames, Proc. of ASCE, Jour. of the EM, No. EM6, pp. 1259-1280, Dec., 1973.

6) Kawahara, M. and K. Horii: Numerical analysis of elastic-plastic framed structures, Proc. of the Symposium on Ultimate Strength of Structures and Their Components, pp. 1325, Sept., 1971.

7) Yoshida, H. and S. Nishida: Lateral-torsional properties of wide-flange sections with residual stresses, Memoirs of the faculty of Technology, Kanazawa University, No. 3, Vol. 6, pp. 147-157, 1971.

8) Ohta, T. and T. Nakazawa: Elasto-plastic analysis of steel pipes subjected to bending and twisting moments (First Report), Technology Reports of Kyushu University, No. 4, Vol. 46, pp. 502-509, Aug., 1973. (in Japanese)

9) Usami, T.: Inelastic behavior of thin-walled open sections under combined axial force, bending and twisting, Proc. of the JSCE, No. 220, Dec., 1973. (in Japanese)

10) Komatsu, S. and T. Sakimoto: Elasto-plastic behavior of steel members under combined torsion and compression, Tech. Report of the Osaka University, Vol. 23, No. 1149, pp. 681-695, 1973.

11) Komatsu, S. and T. Sakimoto: Elasto-plastic behavior of thin-walled steel tubes under combined forces, Proc. of the JSCE, No. 235, pp. 125-136, Mar., 1975.

12) Murray, D. W. and E. L. Wilson: Finite -element postbuckling analysis of thin elastic plates, AIAA Journ., Vol. 7, No. 10, pp. 1915-1920, Oct., 1969.

13) Hofmeister, L. D., G. A. Greenbaum and D. A. Evensen: Large strain, elasto-plastic finite element analysis, Proc. of the AIAA/ ASME, pp. 1248-1254, July, 1971.

14) Sanbongi, S.: Geometrically nonlinear analysis of beam and plate structures including unstable region, collected papers of 5th Conference on Matrix Structural Analysis, pp. 186-193, JSSC, June, 1971. (in Japanese)

15) Stricklin, J. A., W. A. Von Riesman, J. R. Tillerson and W. E. Haisler: Static geo- 
metric and material nonlinear analysis, Paper presented at the 2-nd U.S.-Japan Seminar held in Aug., 1972, Advances in Computational Methods in Structural Mech. and Design, pp. 301-324, UAH press.

16) Nishino, F., Y. Kurakata, A. Hasegawa and T. Okumura: Thin-walled members under axial force, bending and torsion, Proc. of the JSCE, No. 225, pp. 1-15, May, 1974. (in Japanese)

17) for example,

Komatsu, S.: Theory and Calculations of Thin-Walled Structures, Sankaido, pp. 10-14, 1969. (in Japanese)

18) Washizu, K.: Variational Method in Elasticity and Plasticity, Pergamon Press, 1968.

19) for example,

Beaufait, F. W., W. H. Rowan, Jr., P. G. Hoadley and R. M. Hackett: Computer Methods of Structural Analysis, Prentice -Hall Inc., 1970, translated into Japanese by M. Naruoka, Baihukan, 1972.

20) Kao, R.: A comparison of Newton-Raphson methods and incremental procedures for geometrically nonlinear analysis, Computers \& Structures, Vol. 4, pp. 1091-1097, Pergamon Press, 1974.

21) Yamada, Y., N. Yoshimura and T. Sakurai: Plastic stress-strain matrix and its application for the solution of elasto-plastic problems by the finite element method, Int. Journ. of Mechanical Science, Vol. 10, pp. 343-354, 1968.

22) Timoshenko, S. P. and J. M. Gere: Theory of Elastic Stability-Second Edition, Macgraw -Hill, pp. 76-82, 1961.

23) Schulz, D.: Die Traglastberechnung von Planmässig Mittig Belasteten Durchshäben Aus Baushahl unter Berücksightigung von Geometrishen und Structurellen Imperfectionen, Dissertation eingereicht an der Technishen Hochshule in Graz, Juni, 1968.

24) Dadeppo, D. A. and R. Schmidt: Nonlinear analysis of buckling and postbuckling behavior of circular arches, ZAMP, Vol. 20, pp. 847-857, 1969.

25) Harries, H.: Traglasten stählernen Zweigelenklögen mit ausbreiteten Frießzonen, Der Stahlbau, pp. 169-177, 6-1970, pp. 248252, 8-1970.

26) Kuranishi, S. and L. W. Lu: Load carrying capacity of two hinged steel arches, Proc. of JSCE, No. 204, pp. 129-140, Aug., 1972.

27) Heyman, J.: The limit design of space frames, Journal of Applied Mechanics, pp.
157-162, June, 1951.

28) Shinke, T., H. Zui and Y. Namita: Analysis of in-plane elasto-plastic buckling and load carrying capacity of arches, Proc. of JSCE, No. 244, pp. 57-70, Dec., 1975.

29) Namita, Y.: Private communication, March, 1976.

\section{NOTATIONS}

$A \quad=$ cross sectional area;

$A_{e} \quad=$ elastic part of cross sectional area:

$C_{1} \sim C_{6} \quad=$ cross sectional properties defined in Eq. (13);

$D_{1} \sim D_{10} \quad=$ components of matrix $S_{p}$;

$\boldsymbol{d} \quad=$ vector of incremental deformations;

$E \quad=$ Young's modulus of elasticity;

$\boldsymbol{f} \quad=$ vector of incremental internal forces;

$G \quad=$ shear modulus of elasticity;

$I_{\xi}, I_{\eta}, I_{\zeta}=$ moment of inertia of the cross section with respect to $\xi, \eta$ and $\zeta$-axes, respectively;

$I_{\eta \zeta} \quad=$ product of inertia of the cross section;

$\boldsymbol{k}_{e}, \boldsymbol{K}_{e} \quad=$ elastic part of tangent stiffness matrix in local and global coordinates, respectively;

$\boldsymbol{k}_{g}, \boldsymbol{K}_{g} \quad$ =initial stress matrix in local and global coordinates, respectively;

$\boldsymbol{k}_{p}, \boldsymbol{K}_{p} \quad=$ plastic part of tangent stifiness matrix in local and global coordinates, respectively;

$l \quad=$ length of member element;

$M_{\xi}, M_{\eta}, M_{\zeta}=$ stress couples with respect to $\xi, \eta$ and $\zeta$-axes, respectively;

$N \quad$ =axial force;

$O \quad=$ shear center of cross section;

$\boldsymbol{p}, \boldsymbol{P} \quad$ = vector of external loads in local and global coordinates, repectively;

$q \quad=$ shear flow;

$r_{s} \quad=$ distance from the shear center to a tangent drawn at any point on the middle line of the thin wall of the cross section;

$s \quad=$ curvilinear coordinate along the middle line of the thin wall of the cross section;

$S_{\zeta}, S_{\eta} \quad=$ moment of area of the cross section about $\zeta$ and $\eta$ axes, respectively,

$\boldsymbol{S}_{e}, \boldsymbol{S}_{p} \quad=$ elastic part and plastic part of tangent stiffness matrix of the cross section, respectively;

$t \quad=$ thickness of component plates of the cross section;

$\bar{T} \quad=$ force due to twist of longitudinal fibers; 


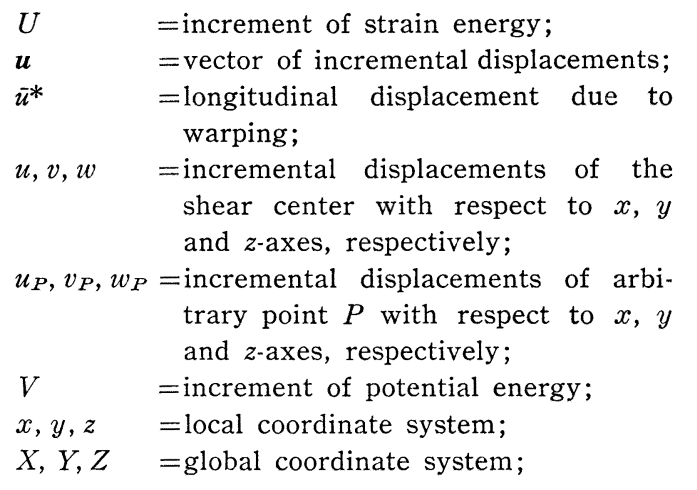
$\bar{\gamma} \quad=$ shear strain;
$=$ normal strain;
$=$ normal strain increment of longi- tudinal axis $x$;
$\theta \quad=$ increment of torsion rate;
$\xi, \eta, \zeta=$ local coordinate values of arbitrary point $P$;
$\pi \quad=$ increment of potential energy;
$\sigma \quad=$ increment of normal stress;
$\tau \quad=$ increment of shear stress;
$\bar{\phi} \quad=$ rotation of cross section;
$\phi_{\eta}, \phi_{\zeta} \quad=$ increments of curvature with respect to $\eta$ and $\zeta$-axes, respectively;

\section{APPENDIX 1}

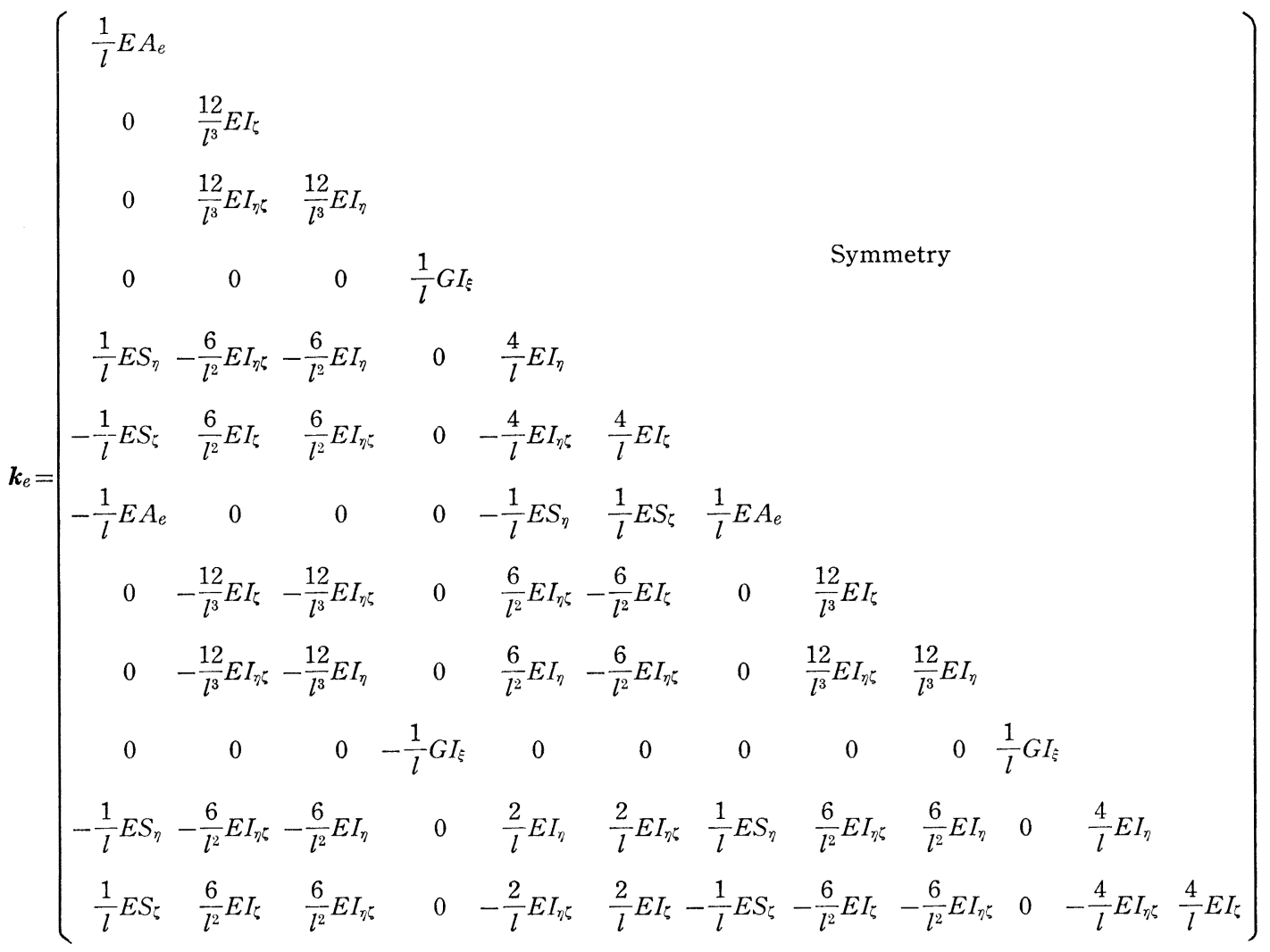

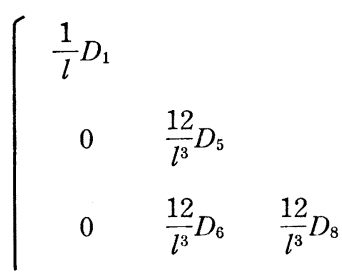

$$
\begin{aligned}
& 7
\end{aligned}
$$


156

S. Komatsu and T. Sakimoto

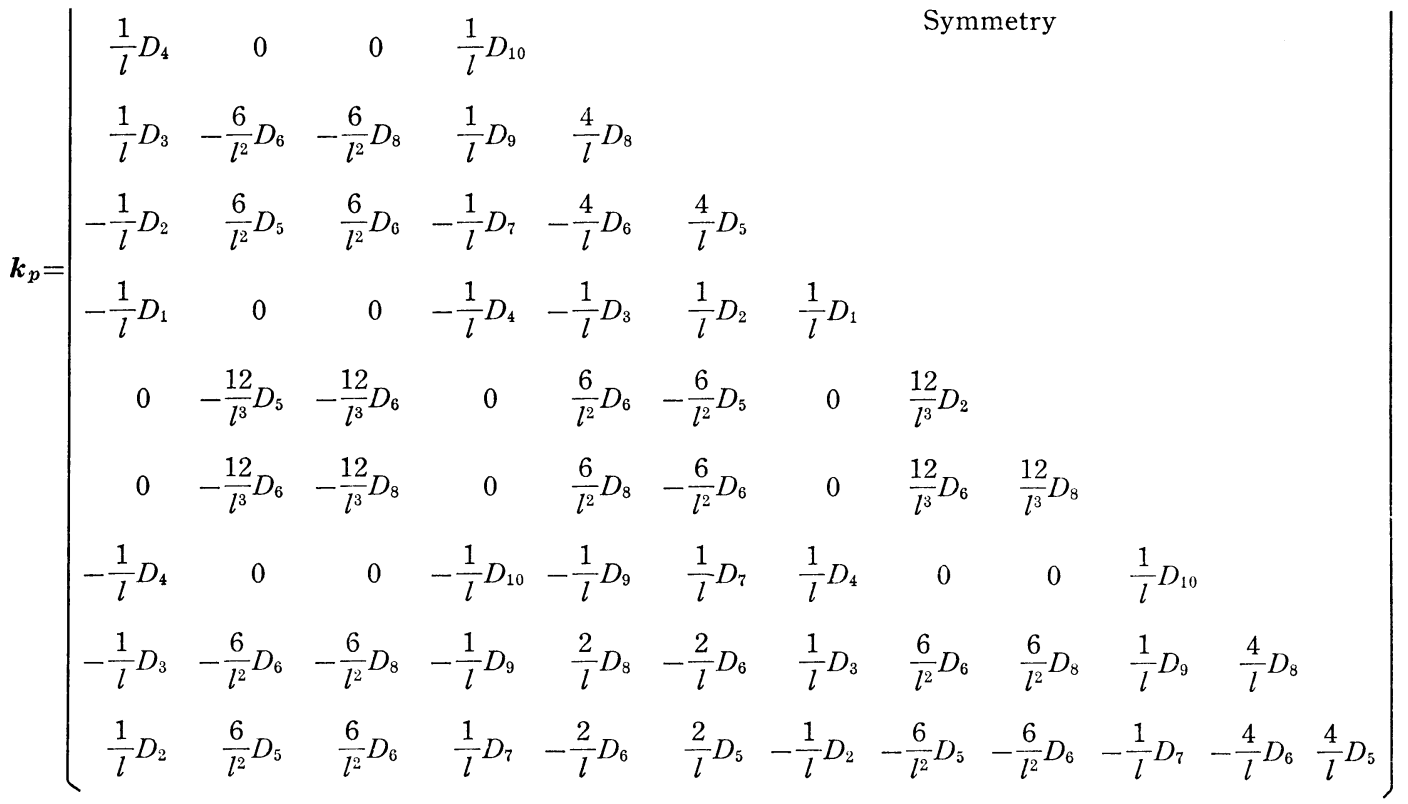

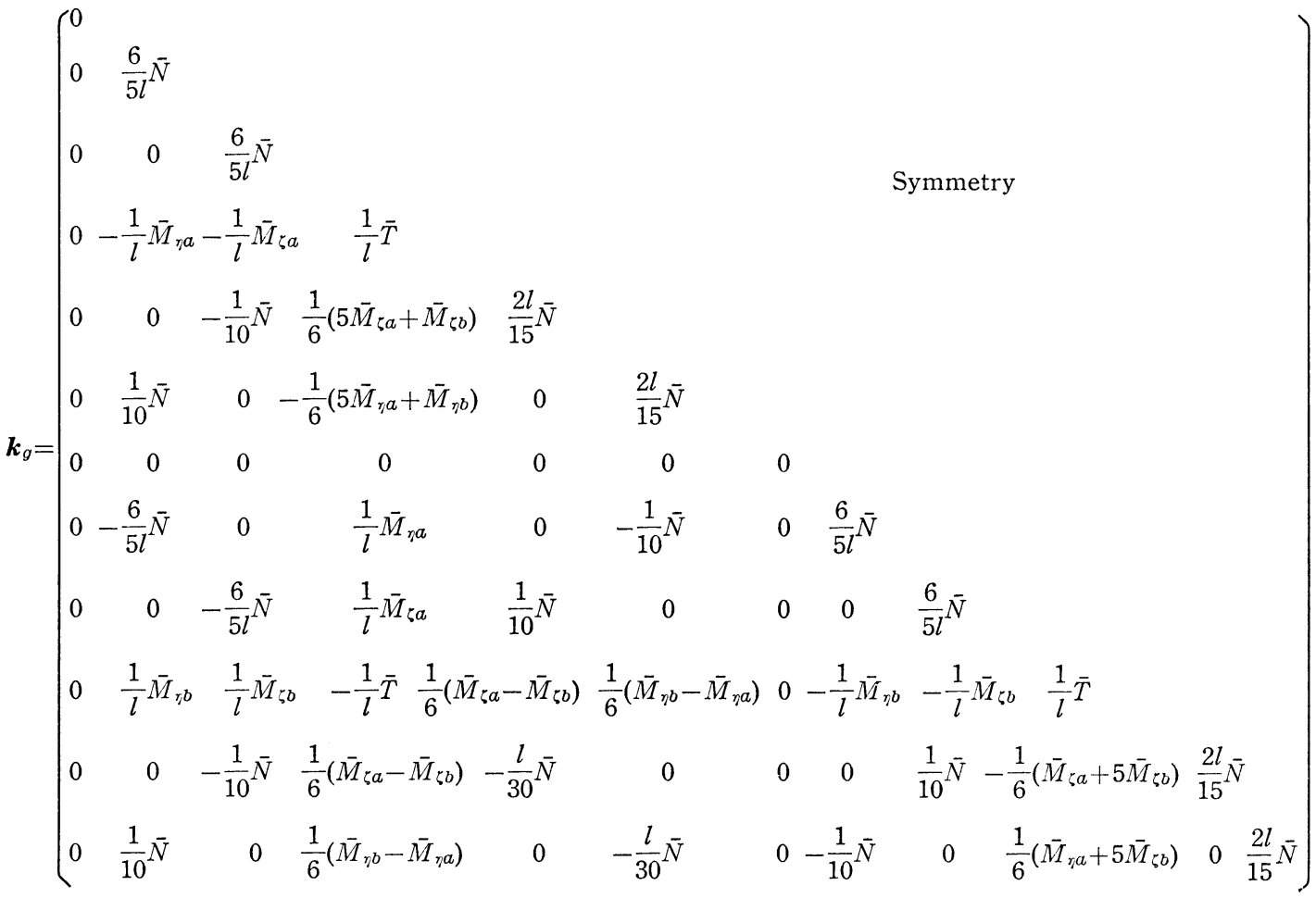

where

$$
\bar{N}=\frac{\bar{N}_{a}+\bar{N}_{b}}{2}, \quad \bar{T}=\frac{\bar{T}_{a}+\bar{T}_{b}}{2} .
$$


APPENDIX 2

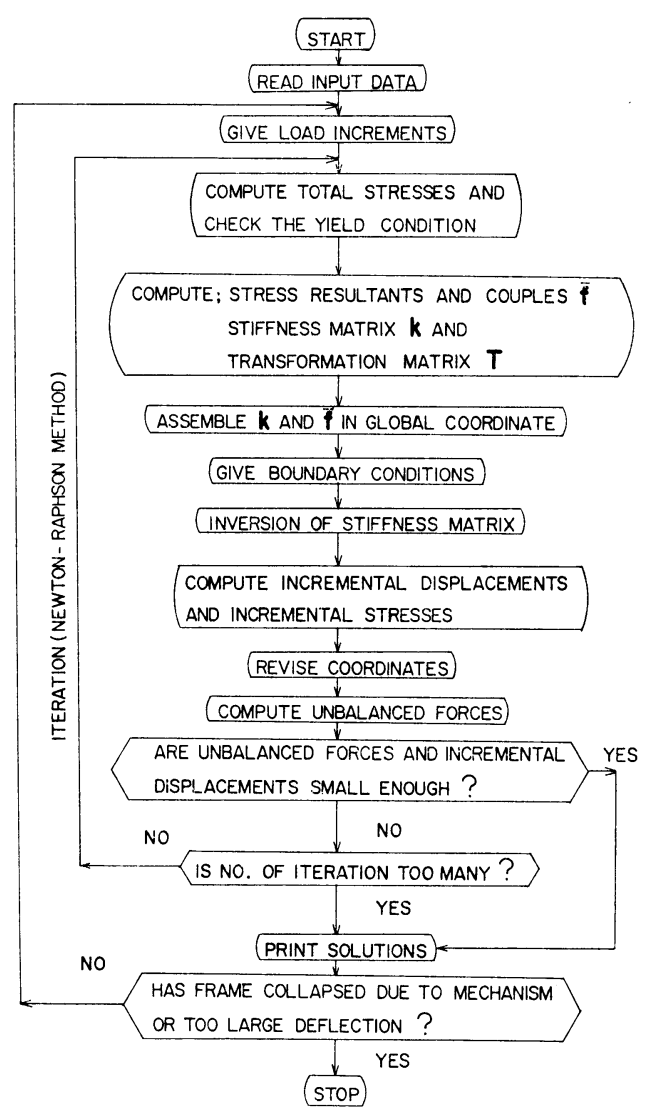

(Received Oct. 28, 1975) 